\title{
Membrane-Grafted Asymmetric Organocatalyst for an Integrated Synthesis-Separation Platform
}

\author{
Christos Didaskalou, ${ }^{\dagger, \#}$ Jozsef Kupai, ${ }^{+, \# \odot ~ L e v e n t e ~ C s e r i, ~}{ }^{\dagger, \oplus \odot}$ Julia Barabas, ${ }^{\S}$ Elemer Vass, ${ }^{\| \odot}$
}

Tibor Holtzl, ${ }^{\S, \perp}$ and Gyorgy Szekely*, ${ }^{*} \odot$

${ }^{\dagger}$ School of Chemical Engineering \& Analytical Science, The University of Manchester, The Mill, Sackville Street, Manchester M13 9PL, United Kingdom

${ }^{\ddagger}$ Department of Organic Chemistry \& Technology, Budapest University of Technology \& Economics, Szent Gellert ter 4, Budapest 1111, Hungary

${ }^{\S}$ Department of Inorganic \& Analytical Chemistry, Budapest University of Technology \& Economics, Szent Gellert ter 4, Budapest 1111, Hungary

"Department of Organic Chemistry, Eotvos Lorand University, Pazmany Peter setany 1/A, Budapest 1117, Hungary

${ }^{\perp}$ Furukawa Electric Institute of Technology, Kesmark utca 28/A, Budapest 1158, Hungary

Supporting Information

ABSTRACT: In this work we introduce a sustainable membrane-based synthesis-separation platform for enantioselective organocatalysis. An azido derivatized cinchona-squaramide bifunctional catalyst was synthesized and subsequently grafted to the surface of a polybenzimidazole-based nanofiltration membrane. The favorable effect of the covalent grafting-due to the change in geometry and increased secondary interactions-on the catalytic activity due to conformational changes was confirmed by quantum chemical calculations. Asymmetric Michael and aza-Michael reactions of 1,3-dicarbonyl and indole, pyrazole, and triazole derivatives to $\beta$-nitrostyrene were performed with as high as $99 \%$ enantiomeric excess. This report on the enantioselective aza-Michael reaction of pyrazoles and

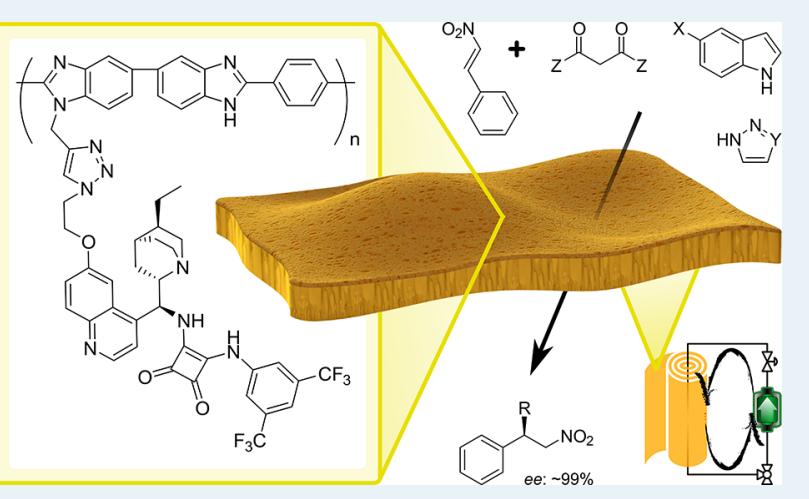
triazoles opens new frontiers in the application of squaramide-based cinchona catalysts. A catalytic membrane cascade reactor was developed for an integrated synthesis-purification process allowing at least $98 \%$ product and substrate recovery, and quantitative in situ solvent recycling. The sustainability of the synthetic methodology was assessed through E-factor and carbon footprint.

KEYWORDS: enantioselective catalysis, polybenzimidazole, recycling, organic solvent nanofiltration, surface modification, Michael addition, catalytic membrane reactor, cinchona-squaramide

\section{INTRODUCTION}

Due to the numerous advantages over conventional transitionmetal catalysis, organocatalytic methodologies have become an attractive synthetic tool in asymmetric catalysis. ${ }^{1,2}$ However, the cumbersome product purification by column chromatography, and the need for catalyst recovery and reuse, call for the design of more flexible and sustainable organocatalytic strategies. Immobilization of organocatalysts on polymer supports was proposed to overcome the separation bottleneck; ${ }^{3,4}$ however, binding a homogeneous catalyst to solid surfaces often led to a deterioration in catalytic activity ${ }^{5}$ and enantioselectivity. ${ }^{6,7}$ Consequently, the scientific and technological challenges of realizing highly efficient asymmetric reactions and simultaneously developing practical separation strategies are yet to be solved. ${ }^{8}$

In the steadily expanding field of organocatalysis, cinchona alkaloids play a prominent role, owing to their high reactivity and enantioselectivity. Chiral squaramides, due to their rigid four-membered ring with electron delocalization, are a promising class of cinchona-based asymmetric organocatalysts for $\mathrm{C}-\mathrm{C}$ bond formation. ${ }^{9}$ Asymmetric Michael and azaMichael reactions of 1,3-dicarbonyl and indole, pyrazole, and triazole derivatives to $\beta$-nitrostyrene were selected as model reactions in this study. The use of the resulting asymmetric products is versatile ranging from medicines to herbicides. ${ }^{10}$

Membrane-based separations, and in particular nanofiltration, have been recognized as sustainable technologies allowing the purification and concentration of chemicals under mild conditions. ${ }^{11,12}$ The surface modification of membranes is a rapidly evolving area with interests from various fields of

Received: May 1, 2018

Revised: July 2, 2018

Published: July 2, 2018 
Scheme 1. Synthesis of the Asymmetric Homo- and Heterogeneous Michael Addition Catalysts ${ }^{a}$
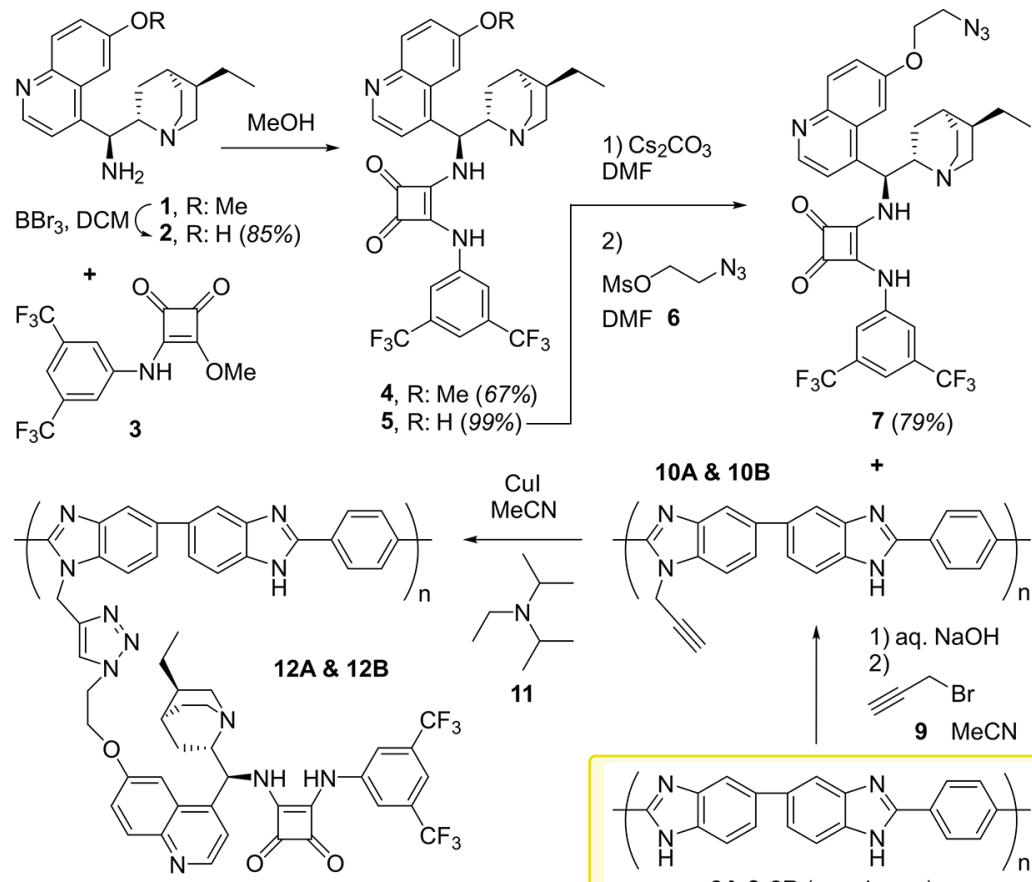

$+$
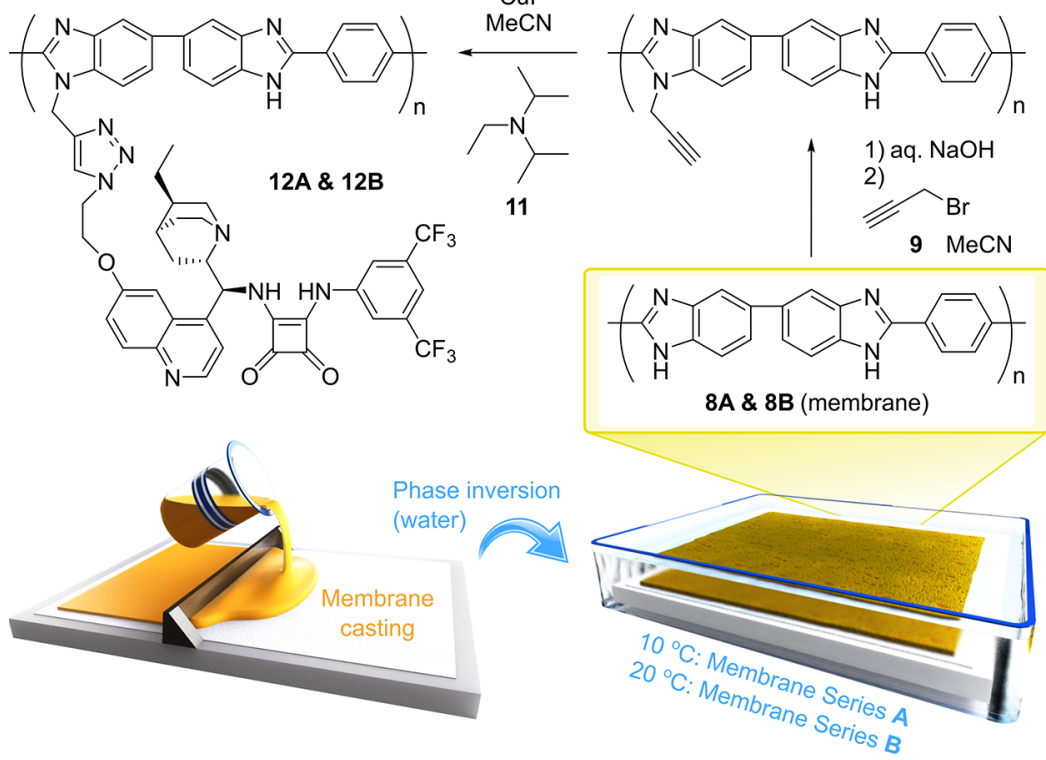

${ }^{a}$ Synthesis of the conventional methoxy (4), and new azidoethylene functionalized (7) cinchona squaramide. The latter was anchored to a propargylated polybenzimidazole (10) via Click chemistry to obtain an organocatalytic nanofiltration membrane (12). Refer to the SI for the synthesis and membrane fabrication procedures.

separation such as desalination, materials, pharmaceuticals, petrochemicals, biomedical devices, and sensors. The membrane surface modification techniques including surface coating, grafting, chemical and plasma treatments, and UV irradiation have recently been reviewed. ${ }^{13}$

Due to the insufficient rejection of catalysts, membraneassisted catalysis requires catalyst enlargement via soluble supports. ${ }^{14}$ The slow leaching of the catalyst or its adsorption to the membrane lead to decreased reaction rate and product contamination. $^{15-17}$ The hypothesis examined in this work is whether covalent grafting of an organocatalyst to a membrane surface would allow the development of a new, sustainable methodology consisting of catalysis, product purification, excess reagent, and solvent recovery in a single unit operation, in addition to overcoming the aforementioned drawbacks. Here we present the new methodology of covalent grafting of organocatalysts on nanofiltration membranes for integrated synthesis-separation.

\section{RESULTS AND DISCUSSION}

Synthesis of Homogeneous and Membrane-Grafted Asymmetric Organocatalysts. The cinchona-squaramide was found to be an excellent catalyst for asymmetric Michael additions. ${ }^{9}$ To graft the catalyst on a membrane, the azido derivative 7, which can be clicked onto any solid support, was synthesized (Scheme 1). Commercially available hydroquinine was converted to cinchona amine derivative $\mathbf{1}$ by reported procedure, ${ }^{18}$ which was demethylated using $\mathrm{BBr}_{3}$ in $\mathrm{DCM}$ to give $2 .^{19}$ Amines $\mathbf{1}$ and $\mathbf{2}$ were reacted with half-squaramide $3^{20}$ resulting in squaramides $4^{21}$ and 5 , respectively. Cinchona squaramide 4 was applied as the benchmark homogeneous organocatalyst. Subsequently, the phenolic hydroxyl group of $\mathbf{5}$ was $O$-alkylated with azidoethyl mesylate $6^{22}$ affording azido derivative 7 .

A polybenzimidazole-based nanofiltration membrane (8) was chosen to anchor the catalyst due to its excellent stability in a wide range of organic solvents. Membrane $\mathbf{8}$ was prepared by casting a $250 \mu \mathrm{m}$ thick film of a $26 \mathrm{wt} \%$ polybenzimidazole (PBI) dope solution in dimethylacetamide solvent, on a nonwoven polypropylene support (Scheme 1). The temperature of the coagulation bath for phase inversion was set at either $10{ }^{\circ} \mathrm{C}$ or $20{ }^{\circ} \mathrm{C}$ resulting in two series of membranes, designated with $\mathrm{A}$ and $\mathrm{B}$, respectively. Lower temperature during the membrane formation results in tighter membranes (Membrane Series A) with low molecular weight cutoff (MWCO). ${ }^{23}$ Membrane 8 was deprotonated with aqueous $\mathrm{NaOH}$ followed by propargyl bromide 9 treatment to form membrane 10 with versatile propargyl groups, which can 
Table 1. Optimization of Membrane Surface Modification on the PBI Monomer Unit 13
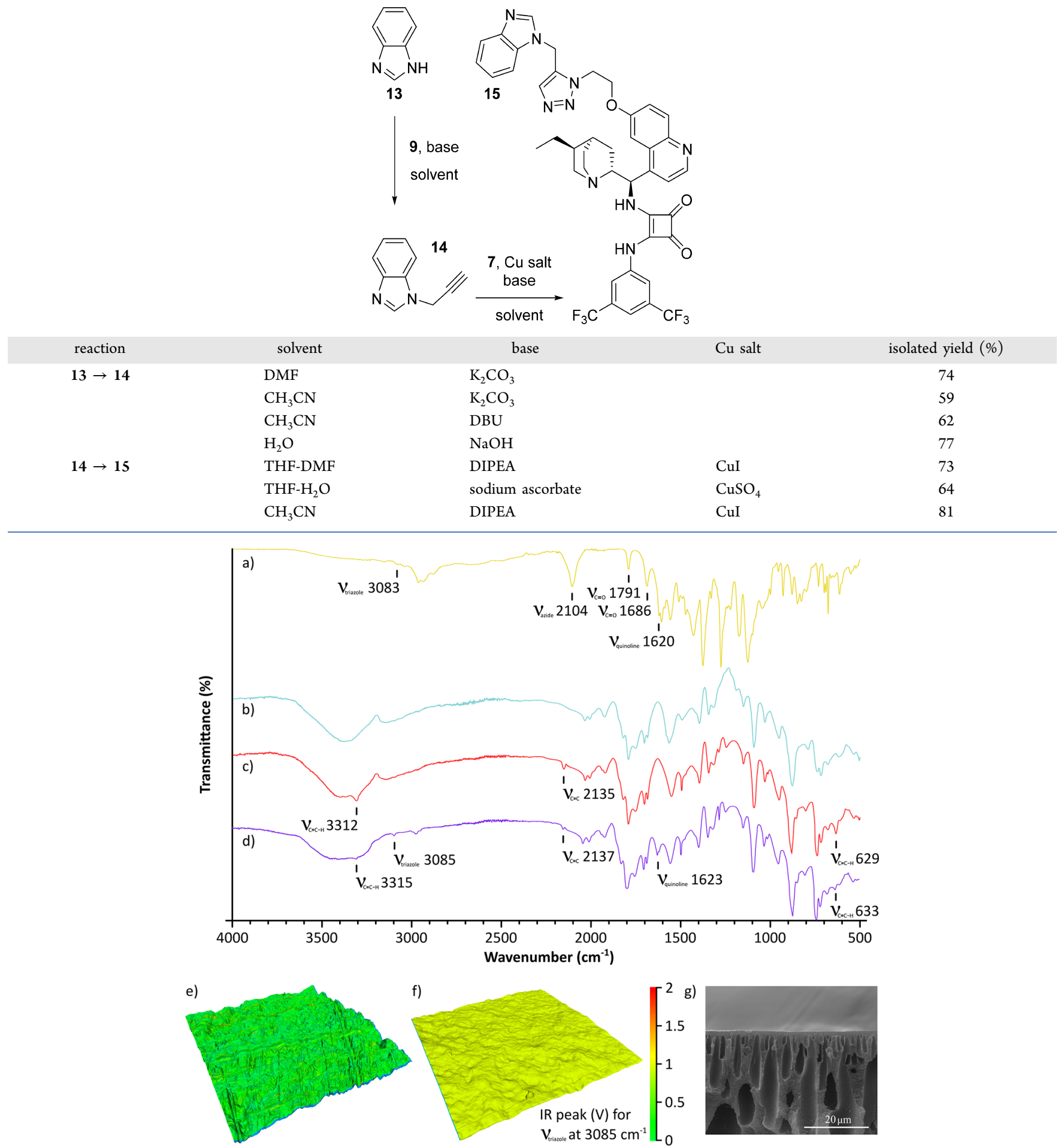

Figure 1. FTIR spectra for compound 7 (a), and membranes 8 (b), 10 (c), and 12 (d). In search of cinchona-squaramide distribution via AFM-IR mapping a $20 \mu \mathrm{m} \times 20 \mu \mathrm{m}$ area of membrane 8 (e) and $\mathbf{1 2}$ (f). The heights and the colors represent the relative topography (AFM) and IR peak intensity, respectively; see the SI for the quantitative $2 \mathrm{D}$ results. The cross-section for membrane 12 was investigated with SEM (g).

readily undergo click reactions. The PBI-anchored catalytic membrane $\mathbf{1 2}$ was prepared in an azide-alkyne cycloaddition from azido derivative 7 and propargylated PBI membrane 10 using copper(I) iodide catalyst and diisopropylethylamine base 11 in acetonitrile. The immobilization reaction was optimized on the monomer subunit 13 of PBI using different solvents, bases and copper salts (Table 1).
The FTIR analysis confirmed the formation and disappearance of the key functional groups during the surface modification of the membranes (Figure $1 \mathrm{a}-\mathrm{d}$ ). The diagnostic stretching vibrations of terminal acetylenic $\mathrm{C}-\mathrm{H}$ and $\mathrm{C} \equiv \mathrm{C}$ bands appeared at $3312 \mathrm{~cm}^{-1}, 2135 \mathrm{~cm}^{-1}$, and $629 \mathrm{~cm}^{-1}$ for the propargylated membrane 10 (Figure 1c). Having clicked the cinchona-squaramide 7 onto the membrane, the character- 
Table 2. Asymmetric Additions of 1,3-Dicarbonyls, Indole, Pyrazole, and Triazole Derivatives to $\beta$-Nitrostyrene 16 Using Homogeneous Catalyst 4 in Different Solvents ${ }^{a}$

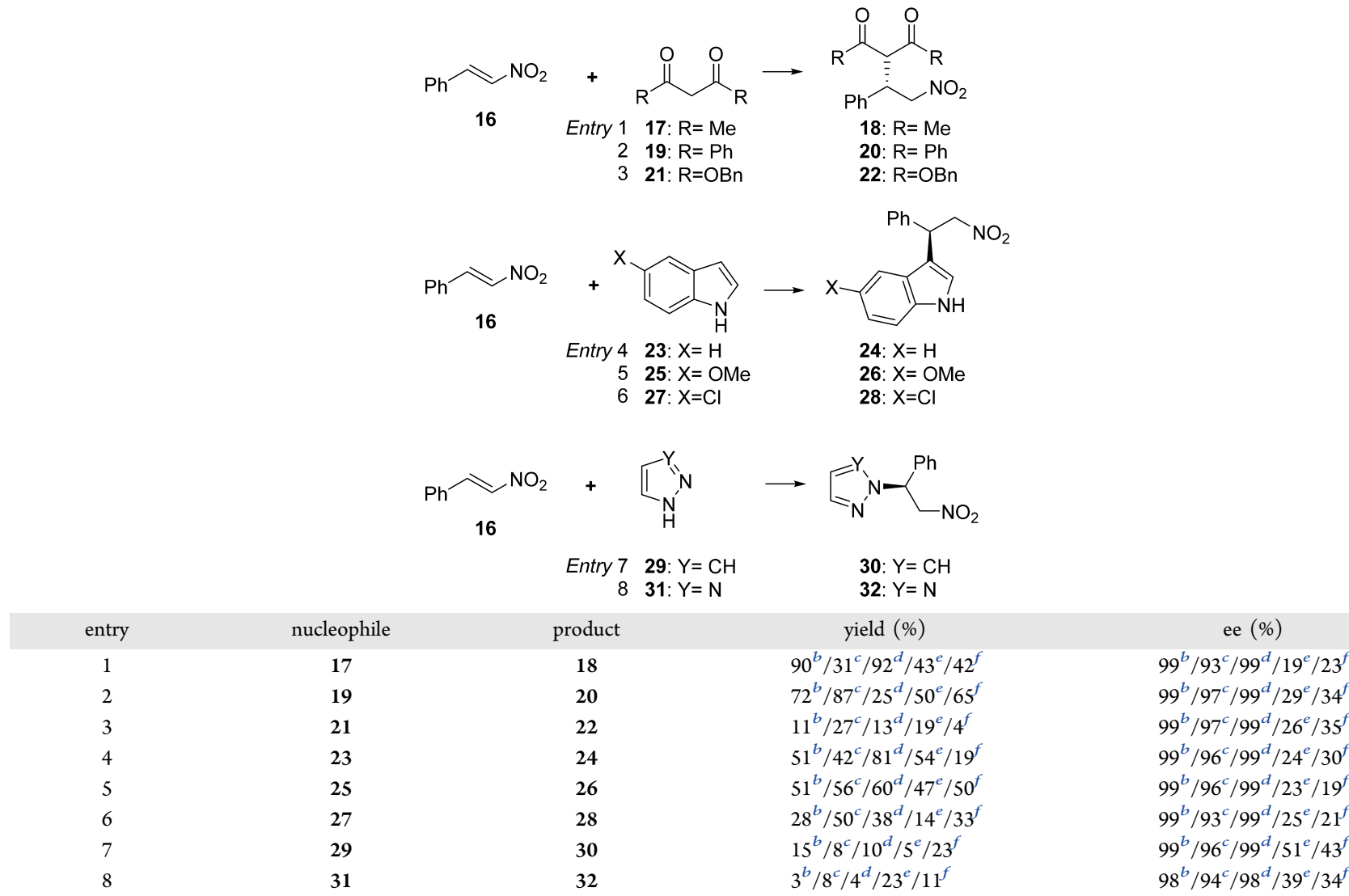

${ }^{a}$ Reaction conditions for entries 1-6: catalyst $4(20 \mathrm{mg}, 31.6 \mu \mathrm{mol})$, nitrostyrene (943 mg, $\left.6.32 \mathrm{mmol}\right)$, nucleophiles (3.16 mmol); reaction conditions for entries 7 and 8: catalyst $4(20 \mathrm{mg}, 31.6 \mu \mathrm{mol}), \beta$-nitrostyrene $16(472 \mathrm{mg}, 3.16 \mathrm{mmol})$, and nucleophiles $(6.32 \mathrm{mmol})$. All the reactions were carried out in $2.0 \mathrm{~mL}$ solvent. ${ }^{b}$ tert-Butyl methyl ether. ${ }^{c}$ Dichloromethane. ${ }^{d}$ Toluene. ${ }^{e}$ Acetonitrile. ${ }^{f}$ Ethyl acetate. The ee values were determined by chiral HPLC.

istic triazole and quinoline peaks appeared at 3085 and 1623 $\mathrm{cm}^{-1}$, respectively (Figure $1 \mathrm{~d}$ ). Quantitative results were obtained using elemental microanalysis, the propargyl/ benzimidazole monomer ratio for membrane 10, and the cinchona-squaramide/benzimidazole monomer ratio for membrane 12 were found to be 0.98 and 0.94 , respectively (see Supporting Information, SI). Excessive washing of the membranes with acetonitrile ensured complete removal of unreacted species. Atomic force microscopy infrared spectroscopy (AFM-IR) revealed (i) uniform distribution of the catalyst on the membrane surface, and (ii) smoother surface as a result of the surface modifications (Figure 1e versus 1f). The cross-section of the membrane shows macrovoids (Figure 1g), which are typical for integrally skinned asymmetric membranes prepared via phase inversion. ${ }^{24}$

Evaluation of the Catalytic Performance. The efficiency of squaramide-based cinchona catalyst 4 in asymmetric transformations was initially assessed through the Michael additions of 1,3-dicarbonyl, indole, pyrazole, and triazole derivatives to $\beta$-nitrostyrene in tert-butyl methyl ether (MTBE), dichloromethane (DCM), toluene, acetonitrile, and ethyl acetate (Table 2). The transformation requires catalyst loadings as low as $1 \mathrm{~mol} \%$, and proceeds at room temperature. MTBE and toluene solvents afforded the products in varying yields (3\% to 92\%) with unprecedented enantioselectivities (98\% to 99\%). Moreover, this is the first report on the enantioselective synthesis of pyrazole (Entry 7) and triazole
(Entry 8) derivatives. The former product was previously synthesized in a racemic form, ${ }^{25}$ however the latter product has not even been reported in a racemic form. Consequently, these results open new directions for the application of squaramidebased cinchona catalysts. The absolute configuration $(S)$ for $\mathbf{3 0}$ and 32 were determined by vibrational circular dichroism (VCD) spectroscopy combined with quantum chemical calculations at density functional theory (DFT) level (see SI).

The thermodynamics of the substrate adsorption was investigated in different solvents (Table 3). The adsorption Gibbs free-energy was found to be negative in all cases, indicating that the adsorption on the membrane-grafted catalyst $\mathbf{1 2}$ is a thermodynamically feasible process. In line with the experimental observations (see Table 2), toluene is the most suitable solvent for the catalysis with the most

Table 3. Thermodynamics of the Substrate Adsorption in Different Solvents

$\begin{array}{cccccc}\text { solvent } & \mathrm{MeCN} & \mathrm{EtOAc} & \mathrm{DCM} & \mathrm{iPr}_{2} \mathrm{O}^{b} & \text { toluene } \\ \Delta G^{a}\left[\mathrm{~kJ} \mathrm{~mol}^{-1}\right] & -46.0 & -49.7 & -50.7 & -54.0 & -64.6\end{array}$

${ }^{a}$ Adsorption Gibbs free-energies (at standard state, concentration of $1 \mathrm{M})$ calculated using the $\omega \mathrm{B} 97 \mathrm{X}-\mathrm{D} / 6-311 \mathrm{G}^{* *}$ method. ${ }^{b} \mathrm{MTBE}$ was estimated with $i \operatorname{Pr}_{2} \mathrm{O}$ as the former solvent is not available in the QChem database. 
negative adsorption Gibbs free-energy among the applied solvents.

The substitution of the homogeneous catalyst 4 with the heterogeneous membrane catalyst $12 \mathrm{~A}$ in a continuous stirredtank reactor resulted in a noticeable decrease in reaction yield (Figure 2). The enantiomeric excess also decreased but still

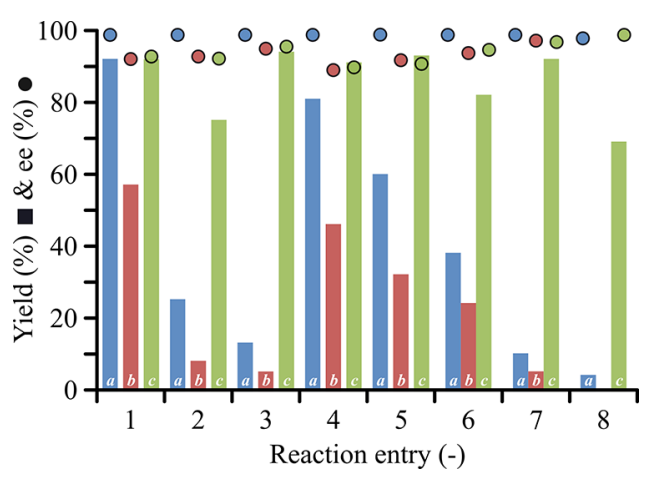

Figure 2. Comparison of catalytic performance for homogeneous catalyst $4^{a}$ in a continuous stirred-tank reactor and heterogeneous membrane catalyst 12A used in either a continuous stirred-tank reactor $^{b}$ or a membrane reactor ${ }^{c}$. The reaction entries refer to the stoichiometry in Table 2. All reactions were carried out in toluene for $24 \mathrm{~h}$. The ee values were determined by chiral HPLC.

remained high in the range of $87 \%$ to $99 \%$. It is speculated that the decrease in yield is a result of the limited mass transport due to the heterogeneous nature of the membrane catalysis. In contrast, the membrane reactor configuration, which involves recirculation of the reaction mixture through membrane catalyst 12A, afforded the products in good to excellent yields (69\% to $94 \%$ ) with excellent enantiomeric excess ( $90 \%$ to $99 \%$ ). For instance, the yield for pyrazole product 22 (Entry 3 ) increased from $5 \%$ to $94 \%$ as a result of improved mass transport. Furthermore, the membrane reactor also out- performed the homogeneous system with regards to yield. In particular, the yield for the triazole product 32 (Entry 8) increased from $4 \%$ to $69 \%$. The enantiomeric excess obtained through membrane catalyst $12 \mathrm{~A}$ remained virtually the same for all entries irrespective of the reactor type, i.e., continuous stirred-tank reactor and membrane reactor.

Design of experiment approach using Modde was used to map the reaction conversion in the membrane reactor, and the results are shown as contour plots in Figure 3. Increasing the reaction time from 6 to $12 \mathrm{~h}$ significantly improved the conversion but longer reaction times are not necessary. Flux is defined as the volume of solvent that permeates the membrane per unit area in a given time, and it is proportional to the applied pressure. Both high pressure and high cross-flow recirculation of the reaction mixture favor the conversion. The correlation between the pressure and the recirculation is reciprocal, i.e. decreasing the pressure necessitates the use of higher recirculation rate. The optimization function of Modde gave $30 \mathrm{bar}, 12 \mathrm{~h}$ and $90 \mathrm{~L} \mathrm{~h}^{-1}$ recirculation flow rate, and these parameters were used in the catalytic membrane reactor.

Catalytic Membrane Cascade Reactor. Solute rejections ${ }^{26}$ for crude mixtures of entry 3 containing 16, 21, and 22, as well as entry 7 containing 29 , and 30 were measured to construct the MWCO curve, which indicates the separation performance of the membranes (Figure 4a). Refer to section 6.2 in the SI for the experimental procedure. The larger the gap in the rejection of the solutes to be separated, the higher the efficiency of the membrane separation. Ideally $100 \%$ rejection is required to obtain $100 \%$ product recovery. The permeance for membrane catalyst $12 \mathrm{~A}$ and $12 \mathrm{~B}$ were found to be $1.01 \pm$ 0.03 and $1.41 \pm 0.07 \mathrm{~L} \mathrm{~m}^{-2} \mathrm{~h}^{-1} \mathrm{bar}^{-1}$, respectively. The lower MWCO of $140 \mathrm{~g} \mathrm{~mol}^{-1}$ (opposed to $200 \mathrm{~g} \mathrm{~mol}^{-1}$ for 12B) and lower permeance for membrane $12 \mathrm{~A}$ originates from the lower fabrication temperature (see Scheme 1). The concept of the catalytic membrane cascade reactor is depicted in Figure $4 \mathrm{~b}$. The first stage (solid lines) was initially operated at 30 bar as a

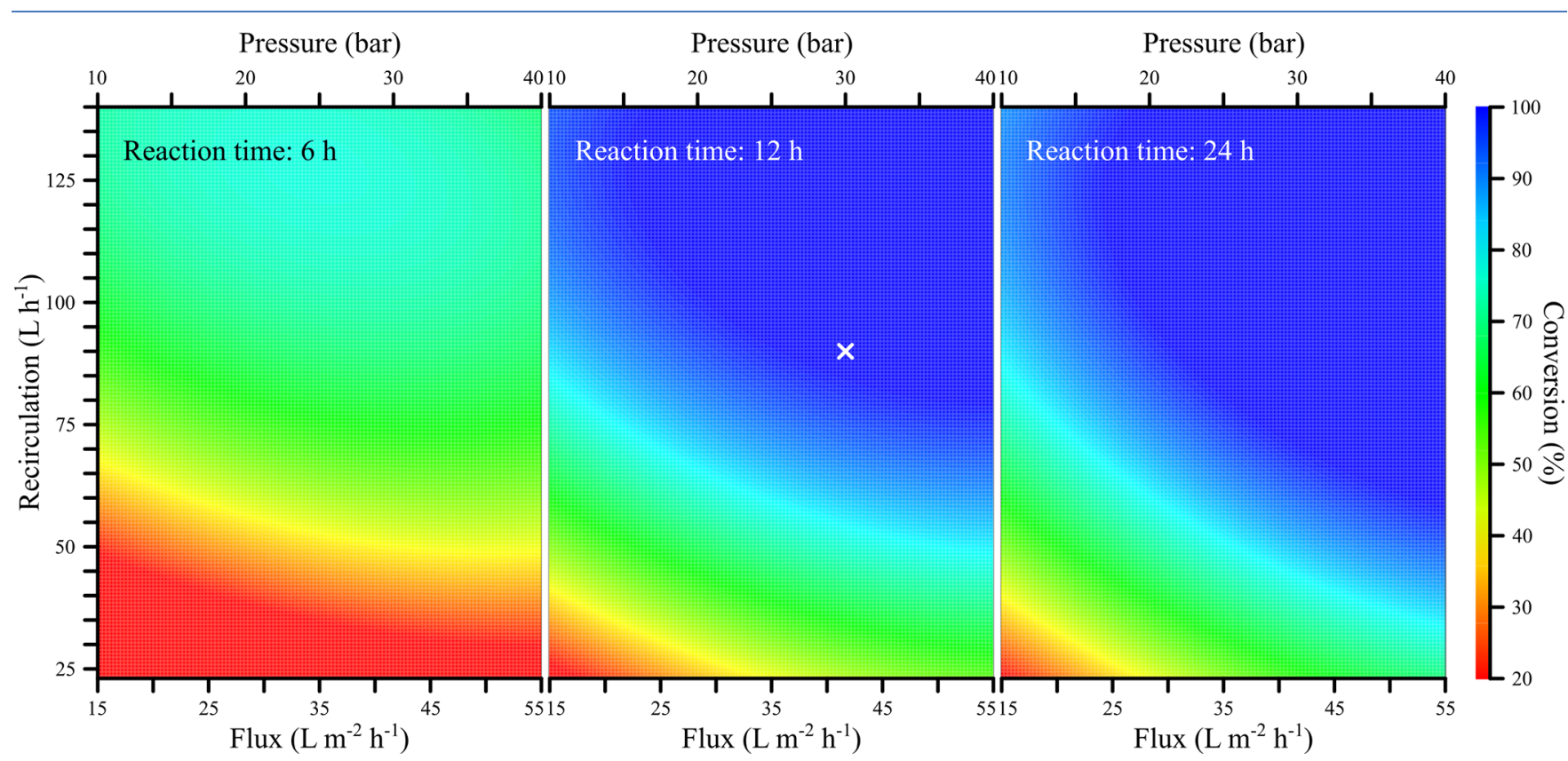

Figure 3. Conversion for product 22 as a function of recirculation flow rate, pressure (or flux) and reaction time obtained from a Central Composite Face design of experiments approach. Catalyst membrane $\mathbf{1 2 A}$ and toluene were used in the membrane reactor. The $\times$ in the design space represents the parameters used for the membrane cascade reactor. 

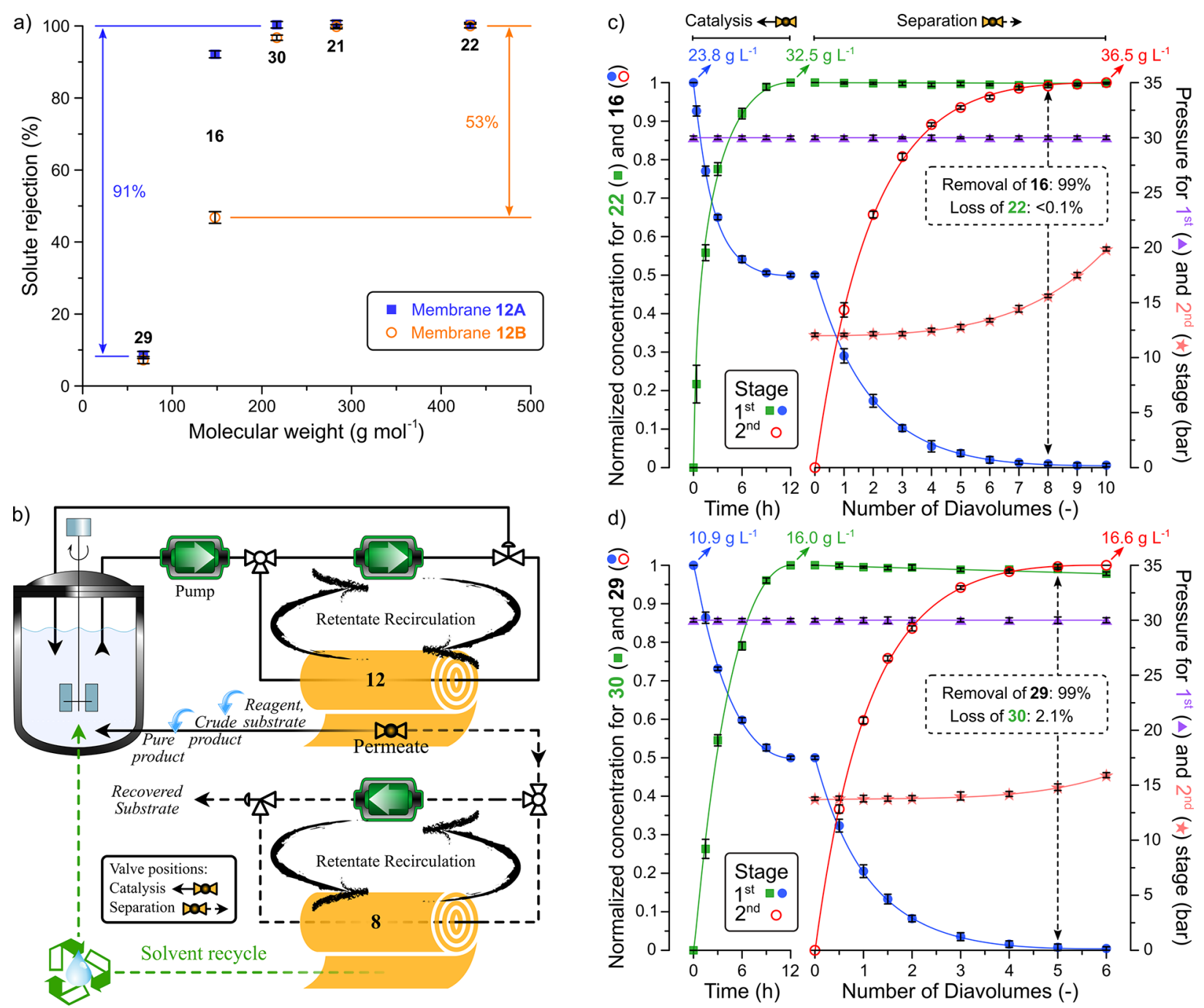

Figure 4. (a) Molecular weight cutoff curves for the membrane catalysts. Reaction mixtures of entry $3(\mathbf{1 6}, \mathbf{2 1}, \mathbf{2 2})$ or entry $7(\mathbf{2 9}, \mathbf{3 0})$ were used to obtain the curves in toluene at 30 bar. The arrows show the rejection difference between the compounds to be separated. (b) Process diagram for the catalytic membrane cascade reactor. The first stage (solid lines) containing membrane catalyst 12 operates as a stand-alone reactor until $100 \%$ conversion is reached. Subsequently, the permeate is directed to the second stage (dashed lines) for separation. Concentration profile for products 22 (c) and 30 (d) in the first stage of the membrane cascade during the catalytic reaction and the separation using catalyst 12A and 12B, respectively.

stand-alone membrane reactor to yield 22 and 30 using membrane catalyst $12 \mathrm{~A}$ and $\mathbf{1 2 B}$, respectively. The concentration profiles for the 12-hour catalysis confirmed 50\% consumption of the reagents $\mathbf{1 6}$ and 29 applied in excess (Figure $4 \mathrm{c}$ and $4 \mathrm{~d}$ ), which corresponds to $100 \%$ conversion.

The catalysis was followed by a two-stage diafiltration, which purified the products in the first stage, while the second stage (dashed lines) simultaneously recovered the unreacted reagents 16 and 29 ( 1 equiv) as well as in situ recycled the solvent. The pressure in the first stage was kept constant at 30 bar. Due to the smaller volume of the second stage, the concentration of reagents increased by about 3 times, which resulted in a 2-8 bar pressure increase (Figure $4 \mathrm{c}$ and $4 \mathrm{~d}$ ). The 8 and 5 diavolumes ${ }^{27}$ allowed $99 \%$ removal of the unreacted reagents, at a cost of $0.1 \%$ and $2.1 \%$ loss of products 22 and 27, respectively (Figure $4 c$ and $4 d$ ). The purification required no addition of solvent due to the in situ solvent recovery unit. The E-factor and carbon footprint for the final process were estimated to be $9.3 \mathrm{~kg} \mathrm{~kg}^{-1}$ and $34.7 \mathrm{~kg} \mathrm{~kg}^{-1}$ after $93 \%$ and $88 \%$ reduction by the second stage, respectively (see
SI). These challenging case studies demonstrated the viability and sustainability of the membrane-grafted asymmetric organocatalysis with integrated synthesis-separation platform. A sensitivity analysis, with regards to solute rejections, product loss and purity, was performed to reveal the robustness and operational window for the proposed methodology (see SI). Owing to the complexity of the process, the continuous operation is a challenge yet to be solved.

Elucidation of Catalyst-Membrane-Substrate Interactions. The possible effect of the covalent grafting on the catalytic performance was investigated using quantum chemical computations. The catalytic membrane is a complex system as the cinchona-squaramide is covalently grafted to the benzimidazole-based polymer. Geometrical structure of the catalyst-membrane-reagent system was investigated using Density Functional Theory (DFT), while the secondary interactions therein were studied using the $\mathrm{XSAPT}(\mathrm{KS})+\mathrm{D}$ method and Non-Covalent Interaction (NCI) analysis (see SI). 

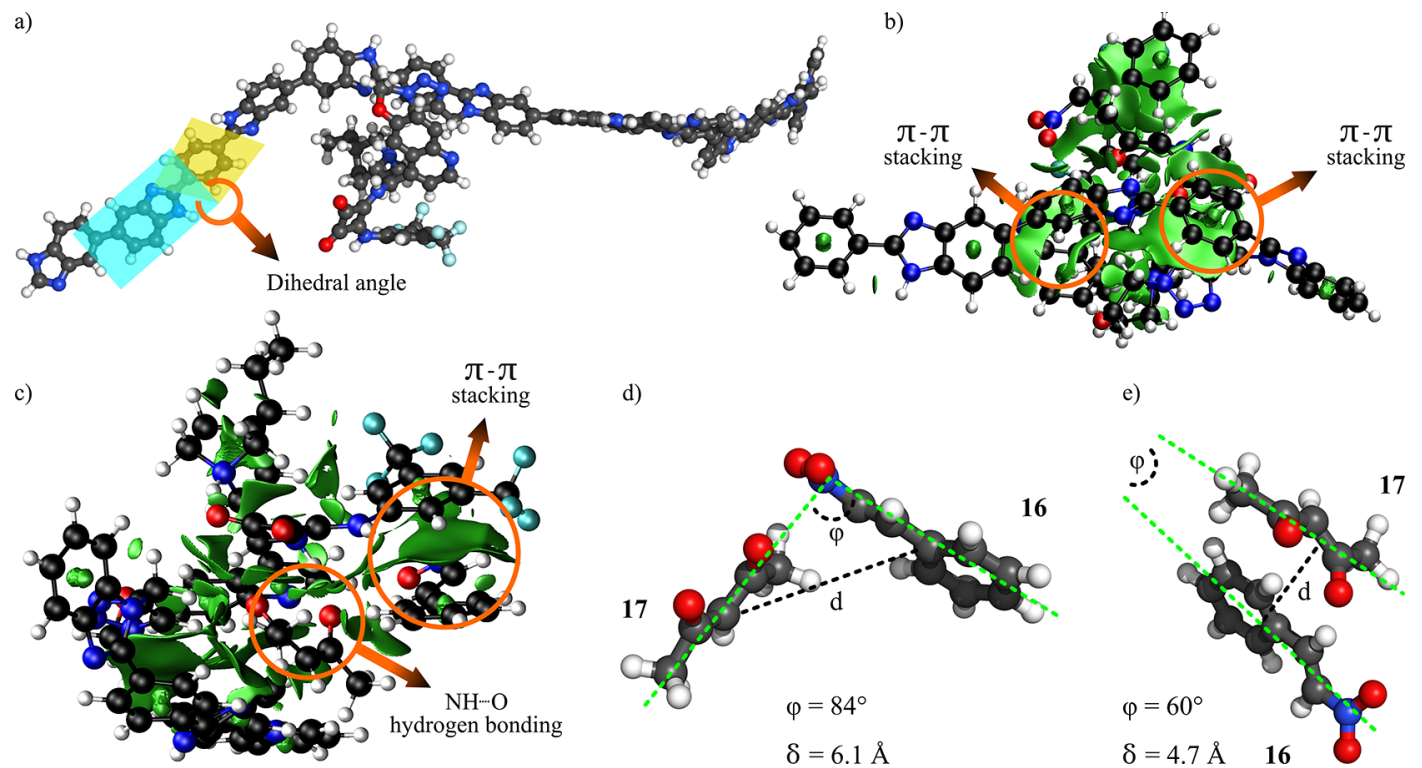

Figure 5. Optimized structure for catalytic membrane 12 (a); the non-covalent interactions (NCI plots) between the polybenzimidazole backbone and the catalyst moiety (b), and between the cinchona-squaramide catalyst moiety and the substrates (c). Plane angle $(\varphi)$ and average distance $(\delta)$ for substrate 16 and reagent 17 during reaction using homogeneous catalyst 4 (d) and membrane-anchored catalyst 12 (e).

Owing to the flexible bonds between the biphenyl rings in the PBI chain, the grafting of the catalyst to the chain may change the conformation of the polymer, which leads to the change of the helicity. The optimized structures (Figure 5a) revealed that the helicity of the catalyst-grafted polymer chain, i.e., dihedral angle between the biphenyl rings, is distorted by $10^{\circ}$ because the benzimidazole and the phenyl rings are not in plane. The NCI analysis concluded that $\pi-\pi$ stacking occurs between the quinoline functionality of the cinchonasquaramide and the benzimidazole units of the PBI, which contributes to the distortion of the polymer chain (Figure $5 b$ ). The participation of the protonated nitrogen of the quinuclidine catalytic site in the catalytic reaction is depressed, while $\pi-\pi$ stacking with the bis(3,5-trifluoromethyl)phenyl group occurs (Figure $5 \mathrm{c}$ ). According to the NCI analysis, there is no secondary interaction between the active moiety of the catalyst (i.e., the squaramide), and the polymer based on the absence of lobes, albeit the grafting resulted in conformation changes. The evolved steric effects explain the breaking of hydrogen bond between the oxo group of the substrate and the amino group of the squaramide. The presence of the membrane increased the secondary interaction between the substrate and reagent during their catalytic reaction from -20 $\mathrm{kJ} \mathrm{mol}{ }^{-1}$ to $-37 \mathrm{~kJ} \mathrm{~mol}^{-1}$ (according to the SAPT calculations), and in parallel decreased both their distance from 6.1 to $4.7 \AA$, and their plane angle from $84^{\circ}$ to $60^{\circ}$. Consequently, the quantum chemical modeling is in line with the observations that the covalent grafting of the catalyst to the membrane could accelerate the catalytic reaction rate.

The hydrophobicity of membranes is an important parameter affecting separation performance. Contact angle measurements, and calculations for solvation Gibbs freeenergies $(\Delta G)$ in water were performed to investigate the effect of surface modification on the hydrophobicity of the membranes (Table 4). The hydrophobicity of the unmodified membrane $\mathbf{8}$ and the propargyl-functionalized membrane $\mathbf{1 0}$ were found to be similar, while the membrane-grafted catalyst 12 showed a distinctive hydrophilic character. More hydro-
Table 4. Calculated and Experimental Membrane Hydrophobicity

$\begin{array}{ccc}\text { membrane } & \Delta G^{a}\left[\mathrm{~kJ} \mathrm{~mol}^{-1}\right] & \text { contact angle }^{b}(\mathrm{deg}) \\ \mathbf{8} & -448 & 78 \pm 3.5 \\ \mathbf{1 0} & -434 & 81 \pm 3.2 \\ \mathbf{1 2} & -508 & 47 \pm 2.7\end{array}$

${ }^{a}$ Solvation Gibbs free-energy calculated using the SM12 model on $\omega \mathrm{B} 97 \mathrm{X}-\mathrm{D} / 6-31 \mathrm{G}^{*}$ level in water. ${ }^{b}$ Average values for three independently prepared membranes.

philic membranes exhibit lower MWCO than their hydrophobic counterparts, which enables effective separation of compounds with relatively low molecular weight. ${ }^{28}$ Moreover, the adsorption of the polar reagents from toluene is more favorable onto hydrophilic surfaces. ${ }^{29}$ This phenomenon facilitates the enrichment of reactants near the catalytic sites on the membrane surface, where the reaction occurs.

\section{CONCLUSIONS}

In summary, we demonstrated an efficient and, given the high yield and enantiomeric excess, eminently suitable membranebased synthesis-separation platform for asymmetric organocatalysis. A cinchona-squaramide and a polybenzimidazole membrane were successfully modified with azide and propargyl functionalities, respectively. The catalyst was successfully clicked onto the nanofiltration membrane. The covalent grafting of the catalyst to the membrane favorable alters the conformation, which enhanced the catalytic activity according to both the quantum chemical calculations and experimental observations. Solvent screening and thermodynamic analysis showed that toluene favors the asymmetric catalysis the most.

Asymmetric reactions between 1,3-dicarbonyls, indole, pyrazole, and triazole derivatives and nitrostyrene were achieved in good to excellent yields (69\% to 94\%) with excellent enantiomeric excess ( $90 \%$ to $99 \%$ ) using membrane catalyst 12. Design of experiments, in particular Central Composite Face, was used to optimize the parameters for the catalytic membrane reactor. 
The asymmetric organocatalysis with integrated separation was successfully demonstrated in a two-stage membrane cascade reactor. The integrated separation allowed at least $98 \%$ product recovery and $99 \%$ unreacted substrate recovery. The demonstrated new synthetic methodology with in situ solvent recovery mitigated the need for solvent addition for purification. The enhanced organocatalyst and compact synthesis-separation design reduced the E-factor and carbon footprint by $93 \%$ and $88 \%$, respectively. The concept of catalyst-grafted membrane cascade reactor presented herein enables new possibilities for fine chemical manufacturing, and further boosts growth in the exciting new era of organocatalysis.

\section{ASSOCIATED CONTENT}

\section{S Supporting Information}

The Supporting Information is available free of charge on the ACS Publications website at DOI: 10.1021/acscatal.8b01706.

Experimental protocols for the syntheses, membrane fabrication, chemical and morphological characterization, and membrane separations; NMR, IR, and MS spectra; HPLC chromatograms; design of experiments; mathematical framework; quantum chemical, sensitivity, and green metrics calculations; MWCO curves; and methods and their references PDF)

\section{AUTHOR INFORMATION}

\section{Corresponding Author}

*Phone: +44 (0) 161306 4366; e-mail: gyorgy.szekely@ manchester.ac.uk (G.S.).

\section{ORCID}

Jozsef Kupai: 0000-0002-4212-4517

Levente Cseri: 0000-0002-3016-5477

Elemer Vass: 0000-0001-8898-3846

Gyorgy Szekely: 0000-0001-9658-2452

\section{Author Contributions}

"These authors equally contributed to this work.

\section{Notes}

The authors declare no competing financial interest.

\section{ACKNOWLEDGMENTS}

The authors would like to express their gratitude to Mr. Gareth David Smith and the MS laboratory (The University of Manchester) for the MS measurements. The authors are grateful to Mr. Fan Fei (The University of Manchester) for the technical assistance with the AFM-IR. The authors wish to thank the Reviewers for their valuable and insightful comments shaping the final version of this article. J.K. is grateful for the Janos Bolyai Research Scholarship of the Hungarian Academy of Sciences. This work was supported by the Biotechnology and Biological Sciences Research Council [BB/L013770/1] and the Central Hungarian Operational Programme (KMOP4.2.1/B-10-2011-0002).

\section{REFERENCES}

(1) Ooi, T. Virtual Issue Posts on Organocatalysis: Design, Applications, and Diversity. ACS Catal. 2015, 5, 6980-6988.

(2) List, B. Introduction: Organocatalysis. Chem. Rev. 2007, 107, 5413-5415.

(3) Lee, J. W.; Mayer-Gall, T.; Opwis, K.; Song, C. E.; Gutmann, J. S.; List, B. Organotextile Catalysis. Science 2013, 341, 1225-1299.
(4) Finelli, F. G.; Miranda, L. S. M.; de Souza, R. O. M. A. Expanding the Roolbox of Asymmetric Organocatalysis by Continuous-Flow Process. Chem. Commun. 2015, 51, 3708-3722.

(5) Rase, H. F. Handbook of Commercial Catalysts: Heterogeneous Catalysts; CRC Press: New York, 2000.

(6) Zhao, L.; Li, Y.; Yu, P.; Han, X.; He, J. Exploration of Dependence of Organo-Catalyzed Enantioselective Michael Addition on the Pore Size of Mesoporous Host. ACS Catal. 2012, 2, 11181126.

(7) Kasaplar, P.; Riente, P.; Hartmann, C.; Pericás, M. A. A Polystyrene-Supported, Highly Recyclable Squaramide Organocatalyst for the Enantioselective Michael Addition of 1,3-Dicarbonyl Compounds to Beta-Nitrostyrenes. Adv. Synth. Catal. 2012, 354, $2905-2910$.

(8) Xu, X.; Cheng, T.; Liu, X.; Xu, J.; Jin, R.; Liu, G. Chiral Squaramide-Functionalized Imidazolium-Based Organic-Inorganic Hybrid Silica Promotes Asymmetric Michael Addition of 1,3Dicarbonyls to Nitroalkenes in Brine. ACS Catal. 2014, 4, 21372142.

(9) Malerich, J. P.; Hagihara, K.; Rawal, V. H. Chiral Squaramide Derivatives are Excellent Hydrogen Bond Donor Catalysts. J. Am. Chem. Soc. 2008, 130, 14416-14417.

(10) Waser, M. Asymmetric Organocatalysis in Natural Product Syntheses; Springer: Wien, 2012.

(11) Sholl, D. S.; Lively, R. P. Seven Chemical Separations to Change the World. Nature 2016, 532, 435-443.

(12) Szekely, G.; Jimenez-Solomon, M. F.; Marchetti, P.; Kim, J. F.; Livingston, A. G. Sustainability Assessment of Organic Solvent Nanofiltration: from Fabrication to Application. Green Chem. 2014, 16, 4440-4473.

(13) Miller, D. J.; Dreyer, D. R.; Bielawski, C. W.; Paul, D. R.; Freeman, B. D. Surface Modification of Water Purification Membranes. Angew. Chem., Int. Ed. 2017, 56, 4662-4711.

(14) Cseri, L.; Fodi, T.; Kupai, J.; Balogh, G. T.; Garforth, A.; Szekely, G. Membrane-Assisted Catalysis in Organic Media. Adv. Mater. Lett. 2017, 8, 1094-1124.

(15) Siew, W. E.; Ates, C.; Merschaert, A.; Livingston, A. G. Efficient and Productive Asymmetric Michael Addition: Development of a Highly Enantioselective Quinidine-Based Organocatalyst for Homogeneous Recycling via Nanofiltration. Green Chem. 2013, 15, 663674

(16) Fahrenwaldt, T.; Großeheilmann, J.; Erben, F.; Kragl, U. Organic Solvent Nanofiltration as a Tool for Separation of QuinineBased Organocatalysts. Org. Process Res. Dev. 2013, 17, 1131-1136.

(17) Großeheilmann, J.; Büttner, H.; Kohrt, C.; Kragl, U.; Werner, T. Recycling of Phosphorus-Based Organocatalysts by Organic Solvent Nanofiltration. ACS Sustainable Chem. Eng. 2015, 3, 28172822.

(18) Vakulya, B.; Varga, S.; Csampai, A.; Soos, T. Highly Enantioselective Conjugate Addition of Nitromethane to Chalcones Using Bifunctional Cinchona Organocatalysts. Org. Lett. 2005, 7, 1967-1969.

(19) Cassani, C.; Martín-Rapún, R.; Arceo, E.; Bravo, F.; Melchiorre, P. Synthesis of 9-Amino(9-deoxy)epi Cinchona Alkaloids, General Chiral Organocatalysts for the Stereoselective Functionalization of Carbonyl Compounds. Nat. Protoc. 2013, 8, 325-344.

(20) Qian, Y.; Ma, G.; Lv, A.; Zhu, H.-L.; Zhao, J.; Rawal, V. H. Squaramide-Catalyzed Enantioselective Friedel-Crafts Reaction of Indoles with Imines. Chem. Commun. 2010, 46, 3004-3006.

(21) Bae, H. Y.; Some, S.; Lee, J. H.; Kim, J.-Y.; Song, M. J.; Lee, S.; Zhang, Y. J.; Song, E. C. Organocatalytic Enantioselective MichaelAddition of Malonic Acid Half-Thioesters to $\beta$-Nitroolefins: From Mimicry of Polyketide Synthases to Scalable Synthesis of $\gamma$-Amino Acids. Adv. Synth. Catal. 2011, 353, 3196-3202.

(22) Hounsou, C.; Margathe, J.-F.; Oueslati, N.; Belhocine, A.; Dupuis, E.; Thomas, C.; Mann, A.; Ilien, B.; Rognan, D.; Trinquet, E.; Hibert, M.; Pin, J.-P.; Bonnet, D.; Durroux, T. Time-Resolved FRET Binding Assay to Investigate Hetero-Oligomer Binding Properties: 
Proof of Concept with Dopamine D1/D3 Heterodimer. ACS Chem. Biol. 2015, 10, 466-474.

(23) Marchetti, P.; Szekely, G.; Livingston, A. G.; Solomon, M. F. J. Molecular Separation with Organic Solvent Nanofiltration: A Critical Review. Chem. Rev. 2014, 114, 10735-10806.

(24) Hung, W.-L.; Wang, D.-M.; Lai, J.-Y.; Chou, S.-C. On the Initiation of Macrovoids in Polymeric Membranes - Effect of Polymer Chain Entanglement. J. Membr. Sci. 2016, 505, 70-81.

(25) Wu, Y.; Wang, J.; Li, P.; Kwong, F. Y. Catalyst-Free Efficient aza-Michael Addition of Azoles to Nitroalkenes. Synlett 2012, 23, $788-790$.

(26) Solute rejection is defined as a ratio of solute concentration in the permeate stream and solute concentration in the retentate stream. (27) The number of diavolumes is time-like parameter, defined as a ratio of the volume of solvent permeated through the membrane and volume of the feed.

(28) Jimenez-Solomon, M. F.; Bhole, Y.; Livingston, A. G. High Flux Hydrophobic Membranes for Organic Solvent Nanofiltration (OSN)-Interfacial Polymerization, Surface Modification and Solvent Activation. J. Membr. Sci. 2013, 434, 193-203.

(29) Paleos, J. Adsorption from Aqueous and Nonaqueous Solutions on Hydrophobic and Hydrophilic High Surface-Area Copolymers. J. Colloid Interface Sci. 1969, 31, 7-18. 